\title{
Return of dual-isotope SPECT myocardial perfusion imaging? Not so fast...
}

\author{
Milena J. Henzlova, $M D{ }^{a}$ and W. Lane Duvall, $M D^{b}$ \\ ${ }^{a}$ Mount Sinai Medical Center (Mount Sinai Heart), New York, NY \\ ${ }^{\mathrm{b}}$ Division of Cardiology (Henry Low Heart Center), Hartford Hospital, Hartford, CT
}

doi:10.1007/s12350-014-0034-y

\section{See related article, pp. 513-522}

Myocardial perfusion imaging remains the most frequently used non-invasive modality for the detection and risk stratification of patients with obstructive epicardial coronary artery disease (CAD). From its inception more than 40 years ago, the mode of stressing, choice of radioactive tracers, and detection technology, have evolved: From exercise stress alone to a choice of four pharmacological stressors, from thallium-201 (Tl201) to technetium- $99 \mathrm{~m}$ (Tc-99m) tracers, from planar to SPECT imaging, from single head to dual-head cameras, from Na-I crystals to CZT solid state cameras. Despite the long track record of diagnostic accuracy, prognostic robustness and ubiquitous availability, continued success for the modality cannot be taken for granted. With competition from the emergence of other non-invasive modalities (stress echocardiography, cardiac CT angiography, cardiac MRI) and with documented changes in the clinical manifestations of $\mathrm{CAD},{ }^{1,2}$ the demands on the safety, efficiency, and convenience of myocardial perfusion imaging continue to grow.

In the long absence of new tracers (Tc-99m-based agents were introduced more than 25 years ago!), the recent advent of solid state high-efficiency SPECT cameras allows for a re-assessment of by now ancient imaging protocols. Obvious and justified criticism of the length of the traditional rest-stress sequence as well as growing awareness of radiation doses to the patients and

Reprint requests: Milena J. Henzlova, MD, Mount Sinai Medical Center (Mount Sinai Heart), New York, NY; milena.henzlova@ mssm.edu

J Nucl Cardiol 2015;22:523-5.

$1071-3581 / \$ 34.00$

Copyright (C) 2014 American Society of Nuclear Cardiology. to the medical personnel can now be addressed with imagination and inventiveness.

One of the novel tested approaches is described in the current issue of the Journal by authors from Grenoble, France. ${ }^{3}$ The authors propose a fast test, which was utilized in 1,336 patients, but analyzed in only 214 patients. Using the GE Discovery NM 530 high-efficiency SPECT camera (GE Healthcare), a mean dose of $82 \mathrm{MBq}(2.2 \mathrm{mCi})$ of Tl-201 was injected at peak stress, 5 minutes stress imaging followed and a mean dose of $314 \mathrm{MBq}(8.5 \mathrm{mCi})$ of Tc-99m was injected after stress imaging and 5 minutes rest imaging followed 2 minutes later. Thus, the whole stress-rest sequence was completed in $22.4 \pm 4$ minutes. Correlation with coronary angiography in 104 patients with high CAD prevalence was good. Normalcy rate in 110 patients with low pretest probability was also high.

A similar protocol and tracer dosage $(2-2.5 \mathrm{mCi}$ of Tl-201 and 8-10 mCi of Tc-99m) was reported in 2009 by Berman et al using a different high-efficiency CZT cardiac camera (D-SPECT, Spectrum Dynamics, Israel) in 374 patients. ${ }^{4}$ Image quality, radiation dose, and study length were analyzed, but not correlation to angiography.

There are pros and cons of both Tl-201 and Tc-99m as myocardial perfusion agents, even though Tc-99m agents are currently used in the vast majority of SPECT myocardial perfusion imaging studies. Tl-201 has a rapid blood clearance half-time ( $<30$ seconds), a high first pass extraction $(>88 \%)$ and better linearity between flow und uptake. ${ }^{5}$ Tc- $99 \mathrm{~m}$ sestamibi, on the other hand, has a longer blood clearance half-time ( $>1$ minute), smaller first pass extraction (50\%-60\%), and clinically non-significant redistribution. ${ }^{6,7}$ These differences provide a higher contrast between regions of normal perfusion and abnormal perfusion using Tl-201 and result in reduced defect size with Tc-99m. ${ }^{8}$ However, higher Tc-99m energy (140 keV of Tc-99m compared to 70-80 keV of Tl-201) and shorter Tc-99m half-life 
(6 hours compared to 72 hours of Tl-201) allow for higher Tc-99m administered doses, higher count studies, better gated images, and for a higher degree of flexibility of imaging protocols (rest-stress, stress-rest, 1- or 2-day studies).

In the original rest Tl-201/stress Tc-99m dualisotope protocol, ${ }^{9}$ the higher energy Tc-99m was lauded for higher quality stress images to optimize assessment of the extent and severity of stress-induced perfusion abnormalities and accurate measurement of left ventricular size and function. Tl-201 was praised for its ability to assess myocardial viability at rest, all the while minimizing the time to perform the study. In the reversed stress Tl-201/rest Tc-99m dual-isotope protocol, Tl-201's stronger relationship between myocardial blood flow and myocardial extraction to improve the ability to detect mild disease which might be missed by Tc- $99 \mathrm{~m}$ is discussed as a strength. With all of the possible individual benefits of the tracers aside, both versions decreased test time as their main contribution, the original version on traditional Na-I cameras and the newer one on high-efficiency cameras. Both sequences, Tl-201 stress/Tc-99 rest and Tl-201 rest/Tc-99m stress allow for delayed (next day) Tl-201 imaging (and viability assessment). The current paper does not explore the potential for viability detection since patients with LVEF $<35 \%$ were excluded from analysis.

The time savings of the proposed protocol comes at the cost of higher radiation exposure to the patients. A 2.5-3.5 mCi rest Tl-201/20-30 mCi stress Tc-99m "old" dual-isotope protocol results in an almost $30 \mathrm{mSv}$ effective dose, while the "new" $2 \mathrm{mCi}$ stress Tl-201/ $8 \mathrm{mCi}$ rest Tc-99m dual-isotope protocol delivers approximately $12 \mathrm{mSv}$. This is similar to the $10-12 \mathrm{mSv}$ effective dose from a traditional $10 \mathrm{mCi}$ rest $/ 30 \mathrm{mCi}$ stress Tc-99m protocol.

The ASNC goal for 2014 is to complete at least $50 \%$ of myocardial perfusion imaging studies with $\leq 9 \mathrm{mSv}$ exposure which this protocol falls short of. Also, even this lowered dual-isotope dose is substantially higher than a $5.8 \mathrm{mSv}$ effective dose achieved using high-efficiency SPECT cameras $(5 \mathrm{mCi}$ rest $/ 15 \mathrm{mCi}$ stress $\mathrm{Tc}-99 \mathrm{~m})$ or $1 / 2$ time $/ 1 / 2$ dose imaging using iterative reconstruction on a traditional dual-head NaI camera. ${ }^{10}$

One way the proposed protocol could be modified, would be to incorporate the possibility of a stress-first protocol. Most large laboratories and the published literature report that at least $70 \%$ of myocardial perfusion imaging studies in patients without known CAD are normal, and the number is even higher in patients referred from the Emergency Department. While the current study does not report how many of the 1,336 patients had normal stress perfusion, the current clinical environment provides a perfect opportunity for stressonly studies. Reviewing the stress Tl-201 images prior to rest injection would be desirable as both the test duration and radiation dose would be decreased. However, only the rest images were gated in this study and the additional Tc-99m dose was needed for the determination of ventricular size and function. The new highefficiency cameras produce count-rich gated images with Tl-201 that are of markedly better quality compared to a traditional Na-I cameras and can provide diagnostic gated information with Tl-201 alone.

The authors use a novel rapid rest acquisition protocol, acquiring Tc- $99 \mathrm{~m}$ images as soon as 2 minutes after Tc-99m injection resulting in significant time savings by eliminating the Guideline specified 3060 minutes delay. This is perhaps a much more interesting development than resurrecting a dual-isotope protocol, as it would be potentially applicable to all Tc$99 \mathrm{~m}$ studies. Appling this rapid rest image acquisition to a low-dose Tc-99m stress-first protocol when rest images were needed would substantially reduce test time. The previous high-efficiency dual-isotope study using the D-SPECT camera also employed a rapid rest image acquisition and reported good or excellent image quality in $98.9 \%$ of rest images. ${ }^{4}$ The majority $(83.7 \%$ of the patients) had none or only minor extracardiac activity in the field of view. This study, however, did not provide comparison within the same patient of early and conventional delayed acquisition.

A previous study of 30 patients using the same GE Discovery camera found that overall image quality was better with fewer uninterpretable studies using conventional delayed imaging. ${ }^{11}$ More importantly, the summed rest score of the rest images obtained at 60 minutes was lower (1.6 vs 3.2) compared to early acquisition (Figure 1). We had a similar experience with immediate post-injection Tc-99m rest images and have subsequently abandoned the practice (unpublished data). The explanation for this discrepancy is unclear, and may be related to continuous Tc-99m uptake early after tracer injection and/or to minimal $\mathrm{Tc}-99 \mathrm{~m}$ redistribution. Given this ambiguity, prior to accepting very early rest image acquisition as standard practice, further studies are warranted.

Simultaneous acquisition of dual-isotope stress and rest data would be another method of speeding the testing time by eliminating one imaging session, and although not explored in the current study, has been already investigated. The solid state CZT cameras show improved energy resolution and with spillover and scatter correction reconstruction, a single imaging procedure may allow for simultaneous multi-tracer acquisition. In a study of 27 patients, Ben-Haim et al demonstrated comparable diagnostic performance and 


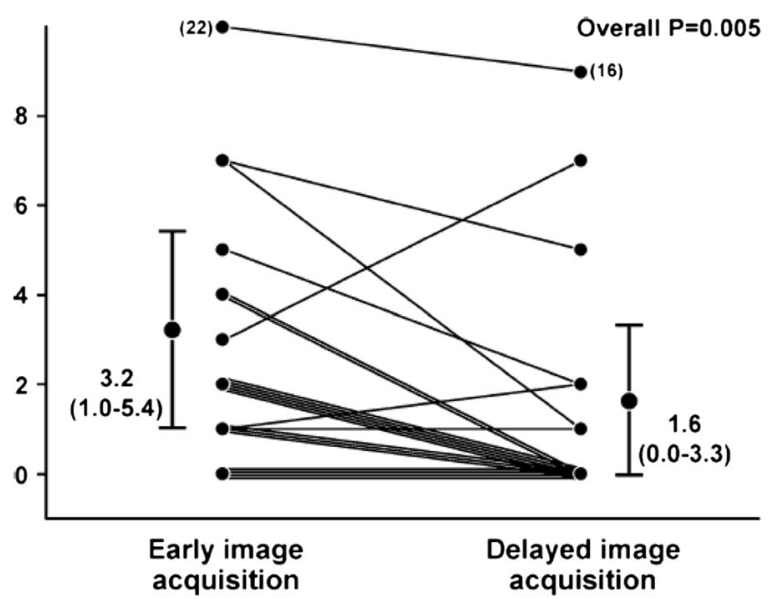

Figure 1. Resting myocardial perfusion as assessed by the summed rest scores of early and delayed image acquisition with the GE Discovery high-efficiency camera in a study by Askew et $\mathrm{al}^{11}$.

image quality of simultaneous and conventional (sequential) acquisition. $^{12}$

The authors of the current paper should be congratulated for their effort to explore and critically assess novel protocols. The proposed dual-isotope protocol perhaps could be recommended in carefully selected subsets of patients: Older patients (perhaps $>70$ years of age, in whom higher radiation dose carries minimal attributable lifetime cancer risk), ${ }^{13}$ or patients with known CAD and/or with a need for detection of myocardial viability (in whom both stress and rest imaging is needed and a 24 hours delayed Tl-201 images may have clinical relevance).

We look forward to future publications in this area.

\section{References}

1. Rozanski A, Gransar H, Hayes SW, Min J, Friedman JD, Thomson LE, et al. Temporal trends in the frequency of inducible myocardial ischemia during cardiac stress testing: 1991 to 2009. J Am Coll Cardiol 2013;61:1054-65.

2. Cheng VY, Berman DS, Rozanski A, Dunning AM, Achenbach S, Al-Mallah M, et al. Performance of the traditional age, sex, and angina typicality-based approach for estimating pretest probability of angiographically significant coronary artery disease in patients undergoing coronary computed tomographic angiography: Results from the multinational coronary $\mathrm{CT}$ angiography evaluation for clinical outcomes: an international multicenter registry (CONFIRM). Circulation 2011;124:2423-32, 1-8.

3. Barone-Rochette G, Leclere M, Calizzano A, Vautrin E, Gallazzini-Crepin C, Broisat A, et al. Stress thallium-201/rest technetium-99m sequential dual-isotope high-speed myocardial perfusion imaging validation versus invasive coronary angiography. J Nucl Cardiol 2014. doi:10.1007/s12350-014-0016-0.

4. Berman DS, Kang X, Tamarappoo B, Wolak A, Hayes SW, Nakazato R, et al. Stress thallium-201/rest technetium-99m sequential dual isotope high-speed myocardial perfusion imaging. JACC Cardiovasc Imaging 2009;2:273-82.

5. Strauss HW, Pitt B. Thallium-201 as a myocardial imaging agent. Semin Nucl Med 1977;7:49-58.

6. Marshall RC, Leidholdt EM Jr, Zhang DY, Barnett CA. Technetium-99m hexakis 2-methoxy-2-isobutyl isonitrile and thallium201 extraction, washout, and retention at varying coronary flow rates in rabbit heart. Circulation 1990;82:998-1007.

7. Primeau M, Taillefer R, Essiambre R, Lambert R, Honos G. Technetium 99m SESTAMIBI myocardial perfusion imaging: Comparison between treadmill, dipyridamole and trans-oesophageal atrial pacing "stress" tests in normal subjects. Eur J Nucl Med 1991;18:247-51.

8. Maublant JC, Marcaggi X, Lusson JR, Boire JY, Cauvin JC, Jacob $\mathrm{P}$, et al. Comparison between thallium-201 and technetium-99m methoxyisobutyl isonitrile defect size in single-photon emission computed tomography at rest, exercise and redistribution in coronary artery disease. Am J Cardiol 1992;69:183-7.

9. Berman DS, Kiat H, Friedman JD, Wang FP, van Train K, Matzer L, et al. Separate acquisition rest thallium-201/stress technetium99m sestamibi dual-isotope myocardial perfusion single-photon emission computed tomography: A clinical validation study. J Am Coll Cardiol 1993;22:1455-64.

10. Duvall WL, Croft LB, Ginsberg ES, Einstein AJ, Guma KA, George $\mathrm{T}$, et al. Reduced isotope dose and imaging time with a high-efficiency CZT SPECT camera. J Nucl Cardiol 2011;18:84757.

11. Askew JW, Miller TD, Ruter RL, Jordan LG, Hodge DO, Gibbons $\mathrm{RJ}$, et al. Early image acquisition using a solid-state cardiac camera for fast myocardial perfusion imaging. J Nucl Cardiol 2011;18:840-6.

12. Ben-Haim S, Kacperski K, Hain S, Van Gramberg D, Hutton BF, Erlandsson K, et al. Simultaneous dual-radionuclide myocardial perfusion imaging with a solid-state dedicated cardiac camera. Eur J Nucl Med Mol Imaging 2010;37:1710-21.

13. Fazel R, Gerber TC, Balter S, Brenner DJ, Carr J, Cerqueira MD, et al. Approaches to enhancing radiation safety in cardiovascular imaging: A scientific statement from the American Heart Association. Circulation 2014;130:1730-48. 